\title{
ESTUDIO DE LA RECUPERACIÓN DEL HIERRO DETRITICO DE LAS ARENAS DE LAS PLAYAS DETACNA
}

\section{RECOVERY STUDY DETRITAL IRON SANDS OF BEACHESTACNA}

\author{
Gualberto Tejada Bedoya ${ }^{1}$; Zenon Sarmiento Mejia ${ }^{2}$; Carlos Huisa Ccori ${ }^{3}$
}

\begin{abstract}
RESUMEN
El trabajo consistió en obtener leyes de hierro existentes en las arenas de playa, luego se dimensionó el volumen de producción de hierro por el método de electromagnetismo, utilizando equipos modernos de alta tecnología que no generan contaminación ambiental, para este propósito se analizó la inversión necesaria y el tipo de financiamiento para poder estimar los costos de operación por tonelada de mineral.
\end{abstract}

Palabras clave: hierro detrítico, electromkagnetismo, arenas de playa

\section{ABSTRACT}

The work was to obtain iron laws existing in the sands of the beach, then iron production volumen was sized by the method of electromagnetism using modern high-tech equipment that do not generate pollution, for this purpose, and analyze the necessary investment the type of financing were analized in order to estimate operating costs per tonne of ore.

Keywords: detrital iron, electromagnetism, beach sands.

\section{I.- INTRODUCCIÓN}

\subsection{Descripción del Problema}

El trabajo consistió en obtener leyes de hierro existentes en las arenas y luego un estudio de mercado de este mineral, desde la producción, comercialización y precios; además se determinó el volumen de producción de hierro por el método de electromagnetismo, utilizando equipos modernos de alta tecnología que no generan contaminación ambiental. Una vez realizados los estudios se evaluó el costo beneficio desde el punto de vista económico, financiero, social y ambiental.

\subsection{Objetivos}

a) Identificar y conocer a detalle las nuevas técnicas, normativas y estudios relacionados con la recuperación del hierro detrítico. b) Contribuir con la acción cognoscitiva de las posibles reservas del mineral de hierro que está distribuido en las arenas de las playas de Tacna.

c) Incentivar la formulación de proyectos de este tipo para otorgar mayores herramientas a los investigadores.

\subsection{Geología de los Depósitos de Arena de Playa}

Son depósitos secundarios derivados de rocas primarias por procesos de intemperismo que, frecuentemente, resultan por enriquecimiento de minerales portadores de hierro. En la mayoría de las veces los depósitos son económicos por tratarse de materiales inconsolidados de explotación y tratamiento relativamente simples. 
Los depósitos de hierro pueden dividirse en:

1. Depósitos de Aluviones de playas y Dunas.

2. Depósitos de áreas de ríos y cascajo.

\section{Depósitos de Aluviones de Playas y Dunas}

Las arenas de las playas son las más importantes en cuanto a contenido de hierro detrítico, producto del intemperismo. Los levantamientos tectónicos muestran que todas aquellas áreas de importancia económica están próximas al continente y se localizan en zonas que en algún tiempo sufrieron un proceso de peneplanización.

Las condiciones de un clima subtropical probablemente favorecen $o$, por lo menos, ayudan a la acción del intemperismo, implicando en la liberación y concentración de los minerales de hierro.

La composición mineral de la mayoría de los depósitos de arena es bien semejante, diferenciándose sólo en el porcentaje de cada mineral presente. Todos los minerales son característicos de gneis, graníticos pegmatíticos.

Estos minerales duros y resistentes al intemperismo son la ilmenita, granate, espinelo, magnetita, estaurolita y epidota.

La historia geológica de los depósitos comerciales de las arenas de playa son semejantes, todos ellos obedecen generalmente a los siguientes pasos:

a) Existencia en el interior del continente de rocas cristalinas donde los minerales pesados son constituyentes accesorios.

b) Un período de peneplanización durante el cual se formó una zona de suelo, liberando los minerales pesados presentes en la roca matriz primitiva.

c) Un surgimiento de una breve erosión de la zona del suelo con una rápida descarga al mar del material erosionado.

d) Algunas veces puede ocurrir un estadio intermedio, desarrollándose un depósito de minerales pesados en la planicie sedimentaria costera, a lo largo de un canal formado por la corriente de transporte, antes que alcance el mineral.

e) Una subsidencia de la costa y formación a lo largo del litoral de bancos de arena, seguida de una acción de las corrientes litorales y olas que llevana las partes más profundas. El material pesado que no es movido con facilidad permanece próximo a su posición original.

f) Una elevación de la costa permite que el ataque de las arenas parcialmente concentradas en los bancos o en los sedimentos de la planicie costera, por las olas, por el viento o por ambos.

Los minerales livianos son llevados por el viento formando las dunas más separadas del mar, dejando los minerales pesados concentrados en las playas.

\section{Depósitos de Arena de ríos y cascajo}

Las arenas y cascajo en las orillas de los ríos contienen acumulaciones de minerales pesados que en algunos lugares son suficientemente ricos en minerales portadores de hierro para ser explotados.

Los depósitos pueden ser limitados por horizontes de arena y cascajo en ellos los minerales pesados están ricamente diseminados. Pueden también constituir lentes en cuerpos irregulares tabulares con algunos centímetros de espesor, estratificados en arenas y cascajo.

La concentración de los más pesados comúnmente ocurre en las partes bajas de valles estrechos, gargantas o en declives próximos de la parte inferior de las rocas que dieron origen a los depósitos.

Los deltas de algunos ríos contienen importantes concentraciones de minerales pesados que son trabajados para el aprovechamiento de hierro.

\section{II.-DISEÑO METODOLÓGICO DE LA INVESTIGACIÓN}

\subsection{Metodología}

\subsubsection{Diseño de Investigación}

- Zona: Arenas de las playas de Tacna.

- Muestra: Calicatas para tomar muestras con fines de ser analizadas en el laboratorio de la Escuela de Metalurgia de la Facultad de Ingeniería y laboratorio Particular.

Se obtuvo un concentrado de hierro detrítico por el método electromagnético. 


\subsubsection{Procesamiento y Análisis de los Datos}

Tabla $\mathbf{N}^{\circ} \mathbf{0 1}$. Análisis cualitativo por espectrometría de emisión

\begin{tabular}{|c|c|c|c|c|c|c|}
\hline \multirow{2}{*}{ Arena } & \multirow{2}{*}{$\begin{array}{c}\text { Elementos } \\
\text { mayores } \\
(+10 \%)\end{array}$} & \multirow{2}{*}{$\begin{array}{c}\text { Elementos } \\
\text { menores } \\
(1 \text { al } 10 \%)\end{array}$} & \multicolumn{3}{|c|}{ Elementos trazas } & \multirow{2}{*}{$\begin{array}{l}\text { Vestigios } \\
\text {-10ppm }\end{array}$} \\
\hline & & & \multicolumn{2}{|c|}{ 1000ppm } & 100ppm & \\
\hline \multirow{7}{*}{$\begin{array}{l}\text { Malla: } 100 \\
\text { Amp: } 0,35\end{array}$} & $\mathrm{Si}$ & $\mathrm{Al}$ & $\mathrm{Mn}$ & $\mathrm{Cu}$ & $\mathrm{Ag}$ & $\mathrm{Au}$ \\
\hline & $\mathrm{Fe}$ & $\mathrm{Mg}$ & & $\mathrm{Na}$ & $\mathrm{Ca}$ & \\
\hline & & $\mathrm{Ti}$ & & $\mathrm{V}$ & $\mathrm{Cr}$ & \\
\hline & & & & $\mathrm{Zr}$ & $\mathrm{Ni}$ & \\
\hline & & & & & $\mathrm{Pb}$ & \\
\hline & & & & & $\mathrm{Sn}$ & \\
\hline & & & & & $\mathrm{Zn}$ & \\
\hline \multirow{7}{*}{$\begin{array}{l}\text { Malla:270 } \\
\text { Amp: 0,35 }\end{array}$} & $\mathrm{Si}$ & $\mathrm{Al}$ & $\mathrm{Mn}$ & $\mathrm{Cu}$ & $\mathrm{Ag}$ & \\
\hline & $\mathrm{Fe}$ & $\mathrm{Mg}$ & & $\mathrm{Na}$ & $\mathrm{Ca}$ & \\
\hline & & $\mathrm{Ti}$ & & $\mathrm{V}$ & $\mathrm{Cr}$ & \\
\hline & & & & W & $\mathrm{Ni}$ & \\
\hline & & & & $\mathrm{Zr}$ & $\mathrm{Pb}$ & \\
\hline & & & & & $\mathrm{Sn}$ & \\
\hline & & & & & $\mathrm{Zn}$ & \\
\hline \multirow{6}{*}{$\begin{array}{c}\text { Malla: } 100 \\
\text { Amp: }+0,35\end{array}$} & $\mathrm{Si}$ & $\mathrm{Al}$ & $\mathrm{Mn}$ & $\mathrm{Cu}$ & $\mathrm{Ag}$ & $\mathrm{Au}$ \\
\hline & $\mathrm{Fe}$ & $\mathrm{Mg}$ & $\mathrm{Zr}$ & $\mathrm{Ca}$ & $\mathrm{Ni}$ & \\
\hline & & $\mathrm{Ti}$ & & $\mathrm{Na}$ & $\mathrm{Zn}$ & \\
\hline & & & & $\mathrm{Pb}$ & & \\
\hline & & & & Sn & & \\
\hline & & & & $\mathrm{V}$ & & \\
\hline \multirow{5}{*}{$\begin{array}{c}\text { Malla: } 270 \\
\text { Amp: }+0,35\end{array}$} & $\mathrm{Si}$ & $\mathrm{Al}$ & $\mathrm{Mn}$ & $\mathrm{Ca}$ & $\mathrm{Ag}$ & $\mathrm{Au}$ \\
\hline & $\mathrm{Fe}$ & $\mathrm{Mg}$ & $\mathrm{Zr}$ & $\mathrm{Cu}$ & $\mathrm{Ni}$ & \\
\hline & & $\mathrm{Ti}$ & & $\mathrm{Na}$ & $\mathrm{Zn}$ & \\
\hline & & & & $\mathrm{Pb}$ & & \\
\hline & & & & $\mathrm{V}$ & & \\
\hline
\end{tabular}

Fuente: Laboratorio de INGEMMET

Tabla $\mathbf{N}^{\mathbf{0}} \mathbf{0 2}$. Distribución magnética de arenas

\begin{tabular}{|c|c|c|c|c|c|}
\hline \multirow{2}{*}{ Malla } & \multirow{2}{*}{$\begin{array}{c}\text { Min. Ferromagnético } \\
\text { +o,0 Amp. }\end{array}$} & \multicolumn{3}{|c|}{ Min. paramagnético } & \multirow{2}{*}{$\begin{array}{c}\text { Min. diamagnético } \\
+\mathbf{0 , 3 5}\end{array}$} \\
\cline { 3 - 5 } & $\mathbf{0 , 0 5 A m p}$ & $\mathbf{0 , 1 5}$ Amp. & 0,35 Amp. & \\
\hline+48 & $42,21 \mathrm{~g}$ & $18,04 \mathrm{~g}$ & $16,11 \mathrm{~g}$ & $16,87 \mathrm{~g}$ & $47,29 \mathrm{~g}$ \\
\hline 100 & $52,20 \mathrm{~g}$ & $14,90 \mathrm{~g}$ & $17,79 \mathrm{~g}$ & $22,45 \mathrm{~g}$ & $47,25 \mathrm{~g}$ \\
\hline 270 & $22,78 \mathrm{~g}$ & $1,67 \mathrm{~g}$ & $2,46 \mathrm{~g}$ & $4,92 \mathrm{~g}$ & $3,45 \mathrm{~g}$ \\
\hline
\end{tabular}

Fuente: Laboratorio de INGEMMET 


\subsubsection{Análisis cuantitativo de hierro}

Análisis total de hierro de la muestra AI - ES (50 gr) compósito de cuatro pozos.

$$
\mathrm{Fe}(\%)=11, \quad \mathrm{Fe}_{2} \mathrm{O}_{2}(\%)=15,73
$$

Análisis de hierro, por mallas, de la muestra AI - ES(100 g.).

$\begin{array}{rrr} & \mathrm{Fe}(\%) & \mathrm{Fe}_{2} \mathrm{O}_{s}(\%) \\ & & \\ \text { Malla 48 (27,46g) } & 6,56 & 9,38 \\ \text { Malla 100 (68,35g) } & 10,84 & 15,50 \\ \text { Malla 270 (3,.99 g) } & 43,19 & 61,75\end{array}$

Análisis de hierro por mallas y considerando los rangos de amperajes $(0,15$ y 0,35$)$.

$\begin{array}{lrlll} & & \mathrm{Fe}(\%) & \mathrm{Fe}_{2} \mathrm{O}_{s}(\%) & \\ & & & \\ \text { Malla } & 48 & 13,80 & 19,73 \\ \text { Malla } & 100 & 16,18 & 23,13 \\ \text { Malla } & 270 & & 13,17 & 50,28\end{array}$

\subsection{Explotación de las Arenas de Playa de Tacna}

Las explotaciones suelen llevarse a cabo en un solo banco, con una profundidad inferior, por lo general, a los $20 \mathrm{~m}$.

\section{III.- RESULTADOS}

En las playas de Tacna, se ha hallado hierro detrítico como producto de la erosión, transporte y acumulación en las arenas de las playas.

La recuperación del Hierro detrítico se ha hecho por electromagnetismo.

En la tabla $\mathrm{N}^{\circ} 01$, se obtuvo en la malla $100 \mathrm{y}$ 0.35 amperios, malla 270 y 0.35 amperios, malla $100 \mathrm{y}+0.35$ amperios, malla $270 \mathrm{y}+0.35$ amperios como elementos mayores $(+10 \%)$ sílice y hierro.

En la tabla $\mathrm{N}^{\circ} \mathrm{O} 2$, se utilizó el tamizado en las mallas $+48,100,270$ y teniendo en consideración las características ferro magnéticas, paramagnéticas y diamagnéticas con diferentes amperajes. Se ha recuperado hierro y otros minerales componentes en las arenas de playas.

En la tabla $\mathrm{N}^{\circ}$ 03, se tiene la cantidad proyectado de material limpio zarandeado en toneladas de hierro detrítico.

\section{IV.-DISCUSIÓN}

En los ensayos de laboratorio se pudo observar la presencia de hierro detrítico y otros elementos que con el estudio detallado puede recuperarse y ser rentables.

Tabla $\mathbf{N}^{\circ}$ 03. Programa de producción a largo plazo

\begin{tabular}{|l|c|c|c|c|c|}
\hline \multicolumn{1}{|c|}{ Producto } & $\mathbf{2 0 1 2}$ & $\mathbf{2 0 1 3}$ & $\mathbf{2 0 1 4}$ & $\mathbf{2 0 1 5}$ & $\mathbf{2 0 1 6}$ \\
\hline Material limpio zarandeado (TM.) & $14.974,2$ & 14974,2 & 14974,2 & 14974,2 & 14974,2 \\
Material desmonte (algas, conchuelas, arena etc.) & 134767,8 & 134767,8 & 194767,8 & 194767,8 & 134767,8 \\
\hline
\end{tabular}

\section{Fuente: Estudio Investigadores}

Cuando las formaciones se encuentran en niveles altos, se utilizan equipos convencionales, como palas, cargadoras de ruedas y volquetes. Sin embargo, es frecuente que los materiales se presenten en contacto con el subálveo o los acuíferos infra yacentes, empleándose entonces otros equipos mineros como son las dragas, las dragalinas o las raspas, dando lugar a la posterior formación de lagunas. Después de haber definido el método aplicable, es necesario establecer el sistema de explotación, que estará constituido por los diferentes equipos de arranque, carga y transporte, en el arranque continuo es posible emplear minadores y, en el transporte continuo, bandas transportadoras convencionales, cintas de alta pendiente, minero ductos, etc. En nuestra explotación se utilizó un sistema de transporte continuo y estacionario.
Utilizando la tecnología de electromagnetismo es factible la recuperación del hierro detrítico de las arenas de las playas de Tacna.

\section{V.- CONCLUSIONES}

Las reservas de arenas con contenido de hierro detrítico en las playas de Tacna son aproximadamente 896000000 millones de toneladas métricas.

El proyecto es viable técnica y económicamente. Existen tecnologías de recuperación de detritus de hierro, de las arenas de playas de Tacna, por lo tanto, se proyecta tener una rentabilidad de 6778 208,3 dólares en 5 años con una capacidad de planta de 83 toneladas métricas por hora. 


\section{REFERENCIAS BIBLIOGRÁFICAS}

- BATEMAN ALAN (2001), Yacimientos minerales, Editorial Omega.

- COllazos CARREÓN JESUS (2005), Inversión y financiamiento de Proyectos. Editorial San Marcos, primera edición, LimaPerú.

- FOLKE KAFKA KIENER (1996), Evaluación Estratégica de Proyectos de Inversión. Universidad del Pacífico.

- GAMERO GONZALES NICKO (2006), Proyectos de Inversión Editorial San Marcos, 1era Edición, Lima- Perú.
- GIRODO ANTONIO CARLOS (1971), Recovery of Minerals from Beaches and Dunes, Colorado School of Mines.

- ORCHE ENRIQUE (2001), Manual de Geología e Investigación de Yacimientos Minerales, U.D. Proyectos E.T.S.I. Minas U.P.M.

\section{Correspondencia:}

Gualberto Tejada Bedoya

Ciuda Universitaria fundo "Los Granados"

Av. Miraflores s/n Tacna-Perú
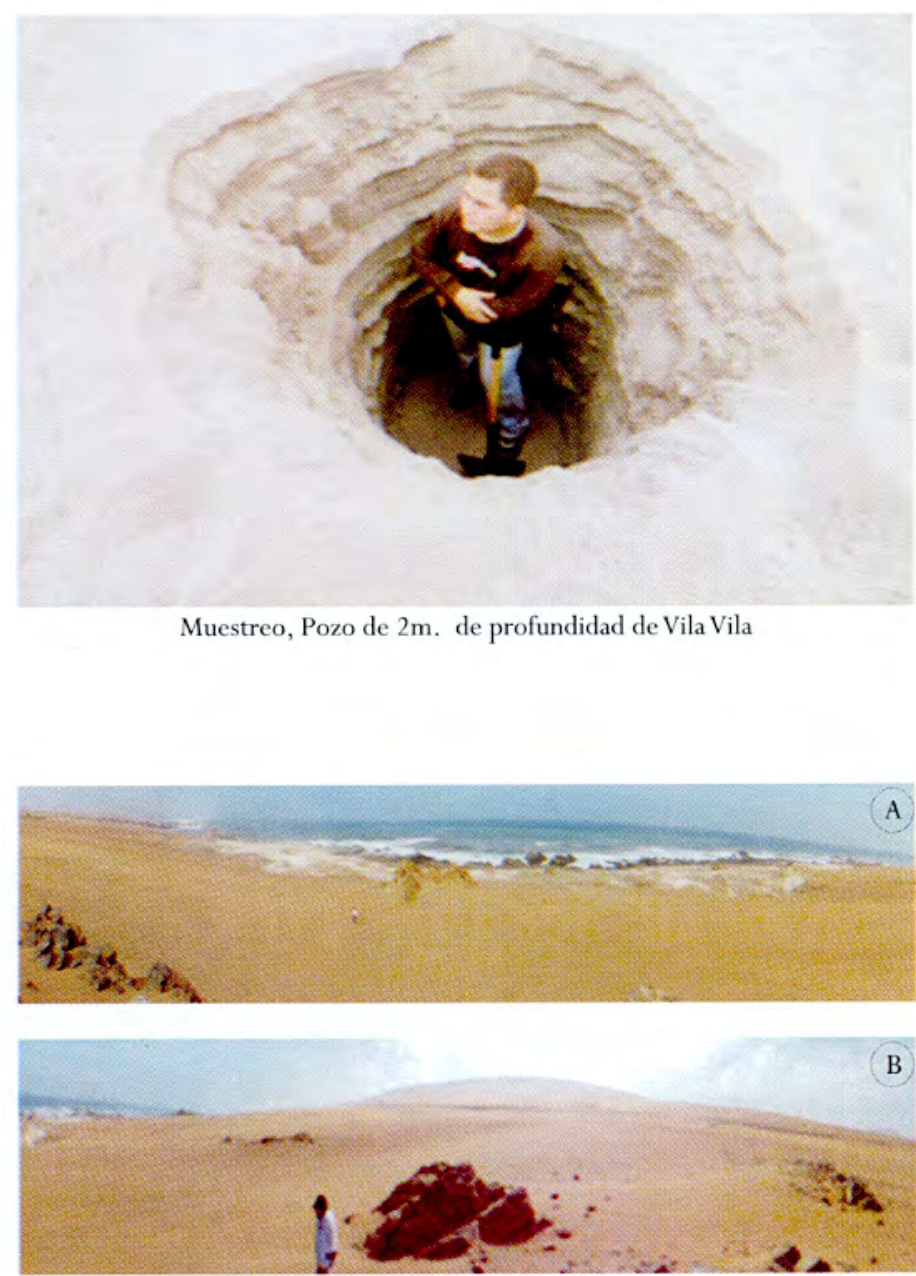

(A), (B) afloramiento de arenas de playa en la zona de Ite 